\title{
Gentest kann Frauen mit Brustkrebs die Chemo ersparen
}

\section{Hier steht eine Anzeige.}

\author{
Ein Rückfallscore auf Basis der Analyse von 21 Genen \\ ermöglicht manchen Frauen mit Brustkrebs den Verzicht \\ auf eine Chemotherapie, obwohl traditionelle Faktoren \\ für ein hohes Rezidivrisiko sprechen.
}

$D_{r}$ ie Westdeutsche Studiengruppe hat neue Ergebnisse der planB-Studie vorgelegt. In dieser prospektiven Phase-III-Studie mit ca. 3.200 Frauen war unter anderem getestet worden, wie sich eine Entscheidung für oder gegen eine Chemotherapie auf der Basis einer Analyse von 21 Genen (Oncotype-DX-Assay) auf die Drei-Jahres-Überlebensraten von Patientinnen mit Hormonrezeptor-positivem und HER2-negativem Brustkrebs auswirkt. Alle Teilnehmerinnen hatten ein erhöhtes Rezidivrisiko mit positiven Lymphknoten oder einem Lymphknoten-negativen Hochrisiko-Tumor (T2, G2 oder G3, hohe uPA/PAI-1 oder ein Alter $<35$ Jahre). Sie wurden einer kompletten Tumorresektion sowie einer Sentinelbiopsie (bei N0-Tumoren) bzw. einer axillären Dissektion (bei positiven Lymphknoten) unterzogen. Für etwa 2.600 Patientinnen lagen Ergebnisse des genetischen Rückfallscores nach dem Oncotype-DX-Verfahren vor. Dieser Test liefert einen Zahlenwert, der das Rückfallrisiko auf einer Skala von 0 bis 100 verortet. Für die planB-Studie wurde das Rückfallrisiko bei Punktzahlen $\leq 11$ als niedrig, bei $12-25$ als intermediär und bei $>25$ Punkten als hoch klassifiziert.

15,3\% der Frauen mit einem Rückfallscore $\leq 11$ bekamen nur eine adjuvante endokrine und keine Chemotherapie. 97,4 \% von ihnen überlebten die folgenden drei Jahre krankheitsfrei - verglichen mit 97,8 \% der Frauen mit intermediärem und 91,9 \% mit hohem Rückfallrisiko unter Chemotherapie. Lymphknotenstatus, Tumorgrad, Proliferationsmaß gemäß Ki-67, Östrogen- sowie Progesteronrezeptorstatus, Tumorgröße und Rückfallscore erwiesen sich in der univariaten Analyse als Prognosefaktoren. Davon verblieben in der multivariaten Analyse Lymphknotenstatus, Grad und Rückfallscore als unabhängige Marker.

Dies sei die erste prospektive Studie zur Chemotherapie bei Brustkrebs, in der die Patientinnen gemäß Rückfallscore behandelt worden seien, so die Autoren. „Frauen mit null bis drei befallenen Lymphknoten, die gemäß traditioneller Parameter Hochrisiko-Patientinnen waren, aber einen Rückfallscore von maximal 11 hatten, zeigten trotz des Wegfalls der adjuvanten Chemotherapie ein exzellentes Drei-Jahres-Überleben.“ Die Diskrepanz zwischen traditionellen prognostischen Markern und dem Rückfallscore unterstreicht laut Autorenteam um Oleg Gluz und Nadia Harbeck die Notwendigkeit einer standardisierten Bewertungsmethode. Zugleich unterstütze sie den möglichen Einbezug gut validierter genetischer Assays zusammen mit klinisch-pathologischen Faktoren in die Entscheidung zur Chemotherapie bei frühem Hormonrezeptor-positivem Brustkrebs. Dr. Robert Bublak

Gluz O et al. West German Study Group Phase III PlanB Trial: First Prospective Outcome Data for the 21-Gene Recurrence Score Assay and Concordance of Prognostic Markers by Central and Local Pathology Assessment. J Clin Oncol 2016, online 29. Februar; doi: 10.1200/JCO.2015.63.5383 